\title{
Ferritin light chain and squamous cell carcinoma antigen I are coreceptors for cellular attachment and entry of hepatitis B virus
}

This article was published in the following Dove Press journal:

International Journal of Nanomedicine

15 February 2012

Number of times this article has been viewed

\author{
Zhaojing $\mathrm{Hao}^{\prime}$ \\ Li Zheng' \\ Lan Kluwe ${ }^{2}$ \\ Weida Huangl,3 \\ 'Department of Biochemistry, School \\ of Life Sciences, Fudan University, \\ Shanghai, People's Republic of China; \\ ${ }^{2}$ Laboratory for Tumor Biology \\ and Developmental Disorders, \\ Department of Maxillofacial Surgery, \\ University Hospital Hamburg- \\ Eppendorf, Germany; ${ }^{3}$ Laboratory \\ for Synthetic Biology, Centers for \\ Nano-Medicine, Shanghai, People's \\ Republic of China
}

\begin{abstract}
Overexpression of squamous cell carcinoma antigen 1 (SCCA1) in hepatitis G2 (HepG2) and Chinese hamster ovary cells can increase hepatitis B virus (HBV) binding capacity by interacting with the preS1 domain of the HBV surface antigen. However, the magnitude of increase in binding capacity was higher by several orders in the former, indicating the existence of additional factor(s) produced by HepG2 cells, which facilitates HBV attachment. Ferritin light chain (FTL) was identified as the sole high hit candidate by screening human liver cDNA library using a bacterial two-hybrid system with either preS or SCCA1 as the bait. Subsequent in vitro protein-protein interaction assays confirmed the binding activity of FTL to both preS and SCCA1, as well as the formation of triple complex preS-FTL-SCCA1, and narrowed down the binding sites on FTL. In vitro overexpression of FTL could further enhance HBV attachment in both HepG2 and Chinese hamster ovary cells, which were already overexpressing SCCA1. Importantly, in vivo co-expression of human FTL and SCCA1 in mouse liver by means of tailvein hydrodynamic injection increased serum levels of HBV surface antigen transiently 24 hours post challenge with HBV-positive human sera, and a large amount of HBV core antigen-positive hepatocytes around blood vessels could be identified by immunohistochemical staining 48 hours post challenge. The data strongly suggest that FTL and SCCA1 may serve as coreceptors in HBV cellular attachment and virus entry into hepatocytes.
\end{abstract}

Keywords: squamous cell carcinoma antigen 1, ferritin light chain, hepatitis B virus, coreceptors

\section{Introduction}

Hepatitis B virus (HBV) is a human hepadnavirus that causes acute and chronic hepatitis and hepatocellular carcinoma. ${ }^{1} \mathrm{HBV}$ infection is considered to be initiated by specific binding of viral envelope proteins to cell membrane structures. Its surface protein antigens (HBsAg) are comprised of three carboxyl-co-terminal HB proteins. ${ }^{2}$ The large surface protein (LHBs) consists of preS1, preS2, and S domains (SHBs), while the middle surface protein consists of the latter two domains. PreS1 domain has a varying length from 109 to 120 amino acids dependent on viral subtypes, while preS2 is a small domain with only 55 amino acids. ${ }^{3}$ Previous studies have suggested a direct involvement of the preS domains (preS1 + preS2) of the LHBs in assisting viral attachment to hepatocytes. ${ }^{4,5}$ The attachment site of LHBs has been functionally narrowed down to amino acids $21-47$ of preS1 by employing synthetic peptides. ${ }^{6,7}$

Several candidates of hepatocyte membrane receptors for HBsAg have been reported, including a membrane receptor for middle surface proteins associated with 
polymerized human serum albumin, ${ }^{8}$ endonexin II (EII) as a specific SHBs binding protein, ${ }^{9}$ and a sialoglycoprotein as a receptor for SHB binding in the uptake of HBV. ${ }^{10,11}$ However, there is little direct evidence that the interaction of $\mathrm{HBV}$ with those receptors could result in viral infections. Recently, a $44 \mathrm{kDa}$ protein has been isolated by affinity chromatography using a tetravalent derivative of the preS1 (21-47) sequence, specifically from detergent-solubilized plasma membranes of the human hepatocarcinoma cell line HepG2. ${ }^{12}$ Sequence analysis identified this protein as the human squamous cell carcinoma antigen 1 (SCCA1), a serine protease inhibitor. ${ }^{13} \mathrm{HBV}$ attachment and internalization in human hepatocytes was prohibited by external recombinant SCCA1, and the prohibition could be recovered by antibodies against recombinant SCCA1 in a dosedependent manner. Overexpression of SCCA1 increased HBV binding capacity both in HepG2 cells and in Chinese hamster ovary (CHO) cells; however, the increase in HBV binding was higher by several orders of magnitude in the former. ${ }^{12}$ This observation implies that additional factor(s) of HepG2 cells could facilitate HBV attachment, and may directly interact with both the cellular SCCA1 and the viral preS domain.

This study aimed to identify such cofactor(s) of SCCA1 for HBV binding. We screened a human liver cDNA library on a bacterial two hybrid system by using both preS and SCCA1 as baits. Ferritin light chain (FTL) was found as a positive match and was further characterized both in vitro and in vivo.

\section{Materials and methods Bacterial two hybrid screening}

BacterioMatch $^{\mathrm{TM}}$ (Stratagene, Santa Clara, CA) was used for two hybrid screening according to the manufacturer's instructions. DNA fragments encoding preS (preS1 + preS2) and SCCA1 were cloned into the EcoRI/BamHI and NotI/XhoI sites of the bait plasmid pBT, respectively. Human liver cell cDNA library in the target plasmid pTRG was purchased from Stratagene (catalog \#240065). The pBT constructs and the pTRG library plasmids were cotransformed into Escherichia coli XL1-Blue MRF' for protein-protein interaction positive clones screening on LB-CTCK plates (LB agar plates supplemented with $300 \mu \mathrm{g} / \mathrm{mL}$ carbenicillin, $15 \mu \mathrm{g} / \mathrm{mL}$ tetracycline, $34 \mu \mathrm{g} /$ $\mathrm{mL}$ chloramphenicol, and $50 \mu \mathrm{g} / \mathrm{mL}$ kanamycin) at $30^{\circ} \mathrm{C}$ for 24 hours. X-gal at a final concentration of $80 \mu \mathrm{g} / \mathrm{mL}$ was used for a second round of screening. For confirmation of the interaction between preS1 and SCCA1, the DNA sequence of SCCA1 was ligated into the pTRG plasmid with NotI/XhoI sites.

\section{Protein-protein interaction assay}

For the in vitro protein-protein interaction study, fusion proteins of preS, FTL, and SCCA1 with glutathione-Stransferase (GST)-tag, maltose binding protein (MBP)-tag, and histidine (His)-tag were expressed in E. coli through pGEX-4T-1 (carrying GST-tag, Amersham Biosciences, Amersham, UK), pMal-c2x (carrying MBP-tag, New England Biolabs, Ipswich, MA), and pET-28a (carrying His-tag, Merck, Darmstadt, Germany), respectively. The corresponding fusion proteins were purified by affinity chromatography with Glutathione Sepharose ${ }^{\mathrm{TM}}$ 4B (GE Healthcare, Little Chalfont, UK), amylose resin (New England Biolabs), and Chelating Sepharose ${ }^{\mathrm{TM}}$ (GE Healthcare), respectively, and were quantified with the bicinchoninic acid protein assay kit (Pierce, Rockford, IL). For a typical pulldown assay, $10 \mu \mathrm{g}$ of a GST-tagged fusion protein was mixed with $10 \mu \mathrm{g}$ of a MBP-tagged fusion protein and $20 \mu \mathrm{L}$ of Glutathione Sepharose 4B or amylose beads in $1 \mathrm{~mL}$ phosphate-buffered saline (PBS, pH 7.0) containing $0.02 \%$ Tween 20. The mixture was incubated for 2 hours at $4^{\circ} \mathrm{C}$ with gentle rotation on a rotating wheel, and the beads were washed thoroughly with PBS containing $0.03 \%$ Tween 20 three times (40 minutes each time) at room temperature. The bound proteins were dissolved by sample buffer for SDSPAGE and subjected to Western blotting assay by anti-MBP monoclonal antibody (NeoMarkers, Fremont, CA), or antiGST polyclonal antibodies (Shenergy Biocolor, Shanghai, China), or anti-His-tag polyclonal antibodies (Santa Cruz Biotechnology, Santa Cruz, CA).

For the in vivo immune precipitation study, fusion proteins of preS, FTL, and SCCA1 with His-tag and HA-tag were expressed in HepG2 cells through pEGFP-N2 (without green fluorescent protein) and pCMV. Following typical immunoprecipitation assay methods, after the precipitation by the His-tag antibody, the bound proteins were inspected by anti-HA-tag antibody (Beyotime, Jiangsu, China).

\section{Cellular attachment experiment}

Infectious HBV patient serum with HBsAg- and HBeAgpositive serology was obtained under informed consent from a chronically infected patient accommodated in Shanghai HuaDong Hospital, China. The HBV titer of this sample was determined to be $6.1 \pm 0.2 \times 10^{6}$ genome equivalents $/ \mathrm{mL}$ by real time polymerase chain reaction. For the attachment experiment, HepG2 and CHO cells cultured in 24-well tissue 
culture plates with Dulbecco's Modified Eagle Medium containing $10 \%$ fetal calf serum, were transfected with pcDNA3.1-based expression vectors pcDNA3.1-SCCA1 or pcDNA3.1-FTL, or both of them, for 24 hours. Then $100 \mu \mathrm{L}$ of HBV patient serum was added to each well, and incubated for 30 minutes for attachment. The cells were washed twice with $0.1 \%$ Tween 20 -containing PBS and twice with PBS alone, and then lysed with $200 \mu \mathrm{L}$ radio immunoprecipitation assay buffer (50 mM Tris-Cl pH $7.4+150 \mathrm{mM} \mathrm{NaCl}+1 \%$ NP-40 detergent $+0.25 \%$ sodium deoxycholate $+1 \mathrm{mM}$ phenylmethylsulfonyl fluoride) at $4^{\circ} \mathrm{C}$. HBsAg dissolved in $100 \mu \mathrm{L}$ radio immunoprecipitation assay buffer was quantified by enzyme-linked immunosorbent assay (Kehua Bio-Engineering, Shanghai, China). The attachment experiment was repeated three times.

\section{In vivo overexpression of human FTL and SCCAI in mouse liver and HBV infection assay}

Six to eight-week-old male mice weighing 18-20 g were used for the experiment. Pure plasmid DNA $(20 \mu \mathrm{g})$ in $2 \mathrm{~mL}$ Ringer's buffer $\left(147 \mathrm{mM} \mathrm{NaCl}+4 \mathrm{mM} \mathrm{KCl}+1.13 \mathrm{mM} \mathrm{CaCl}_{2}\right)$ was injected into mouse tail vein over 5 seconds at a constant speed. This high pressure injection (hydrodynamic injection) delivers the injected material specifically into liver cells of the mice. ${ }^{14,15}$ For the HBV challenge, $10 \mu \mathrm{L}$ of human serum from HBVinfected patients (HBV genome equivalents $6.1 \pm 0.2 \times 10^{6} / \mathrm{mL}$ as determined by real-time polymerase chain reaction) was injected into the mouse tail vein to imitate virus infection 24 hours post hydrodynamic injection. ${ }^{16}$ Determination of HBsAg in serum by enzyme-linked immunosorbent assay, as well as immunohistochemical staining of liver tissues against $\mathrm{HBV}$ core antigen ( $\mathrm{HBcAg}$ ), were carried out at indicated time points post $\mathrm{HBV}$ challenge.

\section{Results \\ Identification of FTL by two hybrid screening}

To search for factors that may interact with both preS and SCCA1, a human liver cDNA library was subjected to bacterial two hybrid screening, using preS or SCCA1 as bait. After two rounds of screening, 101 positive transformants were obtained with preS as bait, and 91 positives were derived with SCCA1 as bait. To our surprise, sequence analysis showed FTL was the only gene overlapped by both groups, with eight hits in preS screening and six hits in SCCA1 screening, respectively.

\section{In vitro and in vivo validation of interaction of FTL with preS and SCCAI}

To examine the interactions between FTL, preS, and SCCA1 in vitro, we prepared recombinant proteins tagged with either GST or MBP epitope to carry out pulldown assays. Using MBP-preS as bait, fusion protein GST-FTL was detected in the eluate whereas GST protein was not, indicating the binding of FTL to preS through FTL/preS interaction. Similarly, pulldown assay by using MBP-SCCA1 as bait confirmed the interaction between FTL and SCCA1 (Figure 1). We also performed Glutathione Sepharose beadsbased pulldown assay and similar results were achieved (data not shown). Furthermore, when HA-tagged preS and SCCA1 were coexpressed with His-tagged FTL protein in HepG2 cells, both tagged preS and SCCA1 were detected in the immunoprecipitation assay by anti-His-tag antibody. These results, combined with two hybrid screening confirmed physical binding between these three proteins.

\section{Characterization of interactive regions on hFTL with preS and hSCCAI}

While SCCA1 was previously characterized as a preSbinding protein, ${ }^{12}$ how FTL binds to preS or SCCA1 was not determined. The three-dimensional structure of FTL contains five $\alpha$ helices, noted as A, B, C, D, and E, respectively. We prepared recombinant GST-tagged mutant fusion proteins, each with a deletion of one of the five helices. GST pulldown assay revealed that the binding motif of FTL to preS was located at $\alpha$-helix A (Figure 2A), because deletion of helix A led to complete loss of binding activity of FTL to preS, whereas deletion of the other four helices did not affect interaction at all. In the case of SCCA1, deletion of helix A did not affect the binding activity of FTL to SCCA1, but deletion of any of the other four helices abolished binding activity. These results suggested differential structural dependence of FTL binding to preS1 and SCCA1, manifested as, if not oversimplified, FTL binding to preS via helix A, and binding to SCCA1 through the other four helices. By this way, FTL, preS, and SCCA1 may interact with each other to form a heterogeneous complex.

To determine the binding motif on preS2 to FTL, we prepared full length and truncated preS2 protein tagged with MBP. Pulldown experiments showed that deletion of eleven amino acids at the N-terminal end of preS2 disrupted interaction between preS2 and FTL, whereas addition of N-terminal eleven amino acids of preS2 restored MBP binding activity to FTL (Figure 2B). 
A

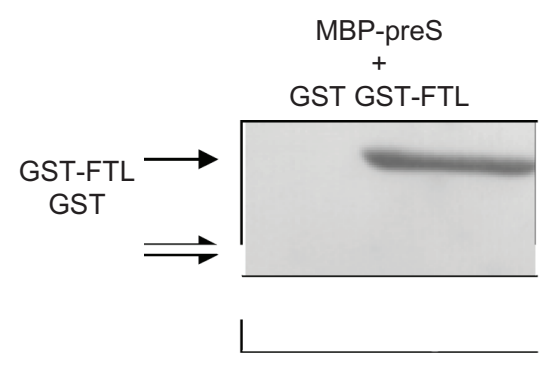

B

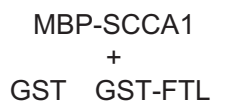

MBP-SCCA1

$+$

GST GST-FTL

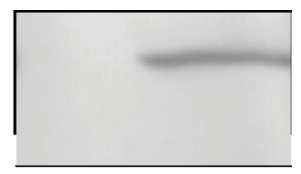

L

C

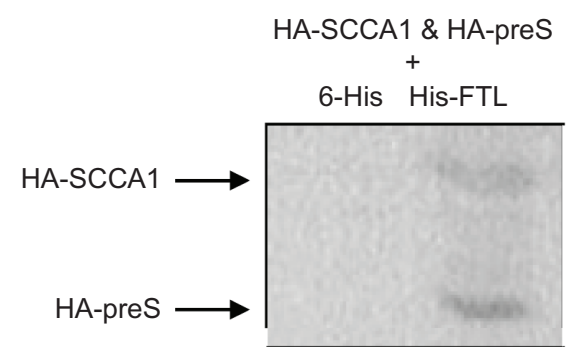

Figure I (A) Western blot of preS-pulldowned proteins; (B) Western blot of SCCAI-pulldowned proteins; (C) Western blot of FTL-pulldowned proteins. For A, MBP-preS was pre-incubated with either GST-FTL or GST before mixing with amylose beads; for B, MBP-SCCAI was pre-incubated with either GST-FTL or GST before mixing with amylose beads; for C, HA-tagged preS and SCCAI were coexpressed with His-tagged FTL protein in HepG2 cells before immunoprecipitation by anti-His-tag antibody. Abbreviations: MBP, maltose binding protein; GST, glutathione-S-transferase; FTL, ferritin light chain; SCCAI, squamous cell carcinoma antigen I.

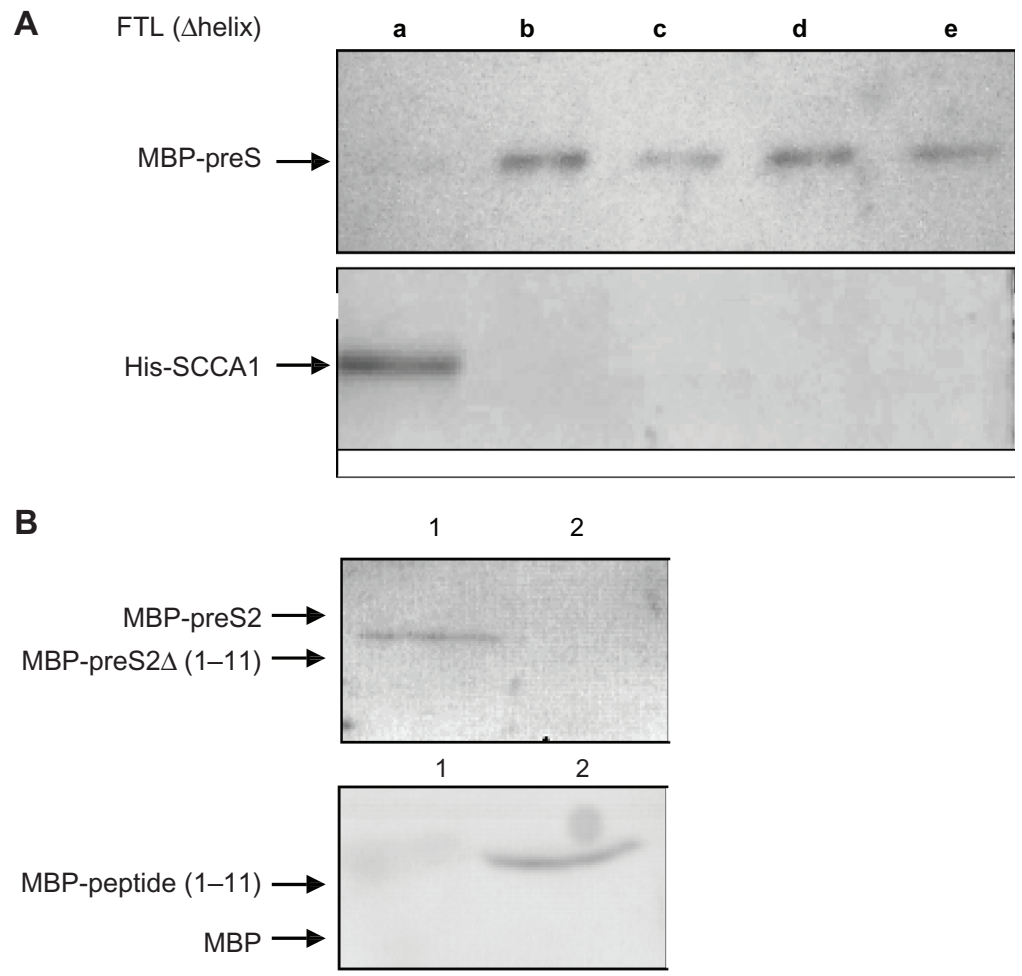

Figure 2 (A) Determination of regions on FTL for interaction with preS and SCCAI. GST-tagged FTL deletion mutant proteins, each with deletion of one of the five $\alpha$-helices (a to e), were allowed to bind to MBP-preS and His-SCCAI, and absorbed with Gutathione-Sepharose ${ }^{\mathrm{TM}}$ beads. Proteins absorbed on Glutathione-Sepharose beads were subjected to Western blotting with anti-MBP antibody (detecting MBP-preS, upper), or with His-tag antisera (detecting His-SCCAI, down). (B) Verification of FTL-binding activity of N-terminal I-I I amino acids of preS2. For the upper, pulldown assay was done by mixing GST-FTL with MBP-preS2 (lane I) or MBP-preS2 $\Delta$ (I-I I) (deletion of $\mathrm{N}$-terminal I-II amino acids of preS2, lane 2), and the proteins recovered by Glutathione-Sepharose beads were subjected to Western blot with anti-MBP antibody. For the down, pulldown assay was done by mixing GST-FTL with MBP (lane I) or MBP-peptide (I-II) (MBP with the peptide of I I amino acids from N-terminus of preS2, lane2), and the proteins recovered by Glutathione-Sepharose beads were subjected to Western blot with anti-MBP antibody.

Abbreviations: FTL, ferritin light chain; MBP, maltose binding protein; SCCAI, squamous cell carcinoma antigen I; GST, glutathione-S-transferase. 


\section{Coexpression of hFTL and hSCCAI in HepG2 cells enhances HBV cellular attachment}

To test whether FTL works as a coreceptor for attachment of HBV on hepatocytes, FTL and SCCA1 were overexpressed in HepG2 cells and viral attachment was measured. As shown in Figure 3, overexpression of SCCA1 in HepG2 enhanced the absorption of HBV significantly, and a further increase was observed when SCCA1 and FTL were coexpressed. However, overexpression of FTL alone showed a remarkable decrease in HBV attachment. Similar data were observed in CHO cells. This phenomenon may be attributed to secreted FTL in culture media which saturated both the binding sites of SCCA1 in cell surface and of preS2 in HBV, by which it blocked the attachment of HBV to HepG2 cells. We tested this hypothesis by adding recombinant human FTL to culture media, and it resulted in reduced attachment of HBV to HepG2 cells as well (data not shown), which is in support of this idea.

\section{Coexpression of hFTL and hSCCAI in mouse liver enhances HBV infection}

To examine if FTL does play a role as a coreceptor of SCCA1 to mediate HBV, human FTL and SCCA1 were expressed in mouse liver using a hydrodynamic injection approach. ${ }^{14,15}$ Mice were injected with a different combination of plasmids to express hFTL, hSCCA1, or both, and a single dose of HBV

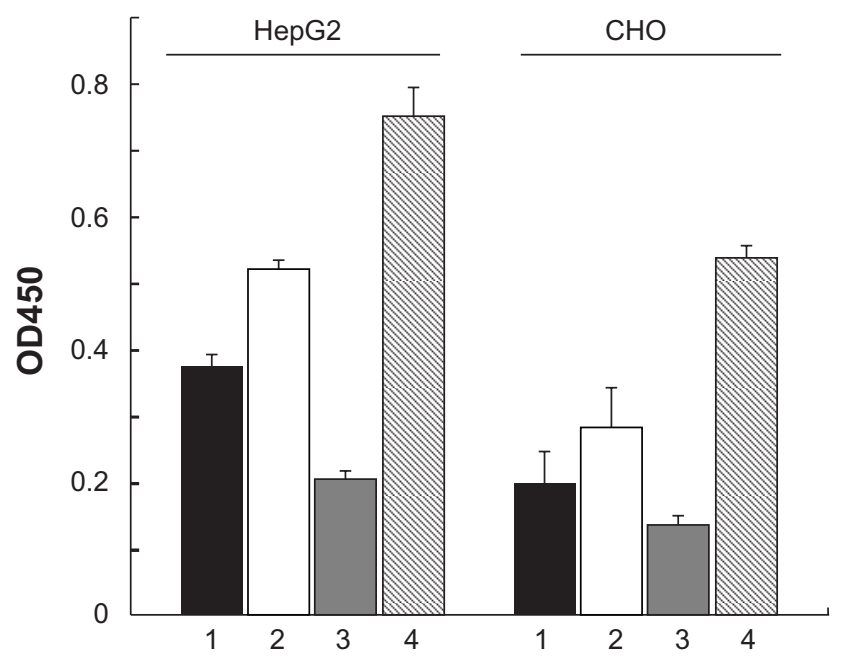

Figure 3 Effect of overexpression of SCCAI, FTL on HBV attachment in HepG2 and $\mathrm{CHO}$ cells. Cells were transfected with pcDNA3.I (column I), pcDNA3.ISCCAI (column 2), pcDNA3.I-FTL (column 3), or both pcDNA3.I-SCCAI and pcDNA3.I-FTL (column 4). HBsAg dissolved in RIPA buffer was measured by ELISA assay to quantify the $\mathrm{HBV}$ attachment experiment.

Abbreviations: $\mathrm{CHO}$, Chinese hamster ovary; SCCAI, squamous cell carcinoma antigen I; FTL, ferritin light chain; $H B V$, hepatitis $B$ virus; $H B s A g$, hepatitis $B$ virus surface protein antigens; RIPA, radio immunoprecipitation assay; ELISA, enzymelinked immunosorbent assay. challenge was administrated 24 hours post hydrodynamic injection. The serum level of HBsAg was measured 48 hours post virus challenge as a readout of virus infection. As shown in Figure 4A, the serum level of HBsAg in mice overexpressing hFTL and hSCCA1 showed a remarkable increase in comparison with the other three groups. Since the increase of serum HBsAg compared to control is likely due to increased synthesis of HBsAg in HBV-infected hepatocytes, this result implied HBV infection and replication in these mice. To confirm this speculation, liver tissues were dissected for $\mathrm{HBcAg}$ immunohistochemical staining 48 hours post $\mathrm{HBV}$ challenge. As shown in Figure 4B, a considerable amount of $\mathrm{HBcAg}$ positive hepatocytes around blood vessels (the binucleated cells as enlarged in Figure 4B (d) inset) could be identified in the group coexpressing FTL and SCCA1. The liver tissues of the other three groups were also extensively investigated, however, no obvious positive staining was detected. The data in Figure 4B strongly suggest that human SCCA1 or human FTL alone was not sufficient to introduce HBV viral particles into mouse liver cells. Only when both of them were expressed in mouse hepatocytes could the HBV viral particles enter mouse hepatocytes. Since the plasmid DNA introduced into hepatocytes by hydrodynamic injection was principally concentrated around liver blood vessels, ${ }^{31}$ this distribution of positive cells may resemble the situation of first round infection of viral particles introduced by tail-vein injection of patient serum.

\section{Discussion}

HBV infection targets hepatocytes in the liver. Interaction of viral proteins and host receptors was believed to be involved in HBV tropism. Characterization of HBV receptor proteins of human hepatocytes has received substantial attention over many years. Many proteins have been reported to show binding activities to the viral surface protein $\mathrm{HBsAg}$, including human interleukin- $6,{ }^{17}$ human immunoglobulin $\mathrm{A},{ }^{18}$ an $80 \mathrm{kDa}$ protein, ${ }^{19}$ asialoglycoprotein receptor, ${ }^{10,11}$ and poly human serum albumin. ${ }^{8,20}$ However, convincing evidence was absent to prove involvement of those candidates in HBV attachment and entry into human hepatocytes. Previous studies have revealed the possibility of the membrane protein SCCA1 as a receptor of HBV, since it was purified by affinity chromatography using a tetravalent derivative of the preS1 (21-47) sequence. ${ }^{12}$ Expression of SCCA1 in mouse liver could extend the retention of HBV in mouse liver. ${ }^{21}$ We started to search for a second factor or factors other than SCCA1 because of the different affinity of HBV to HepG2 and CHO cells which were overexpressing SCCA1. FTL was also the only target which was positive 
A
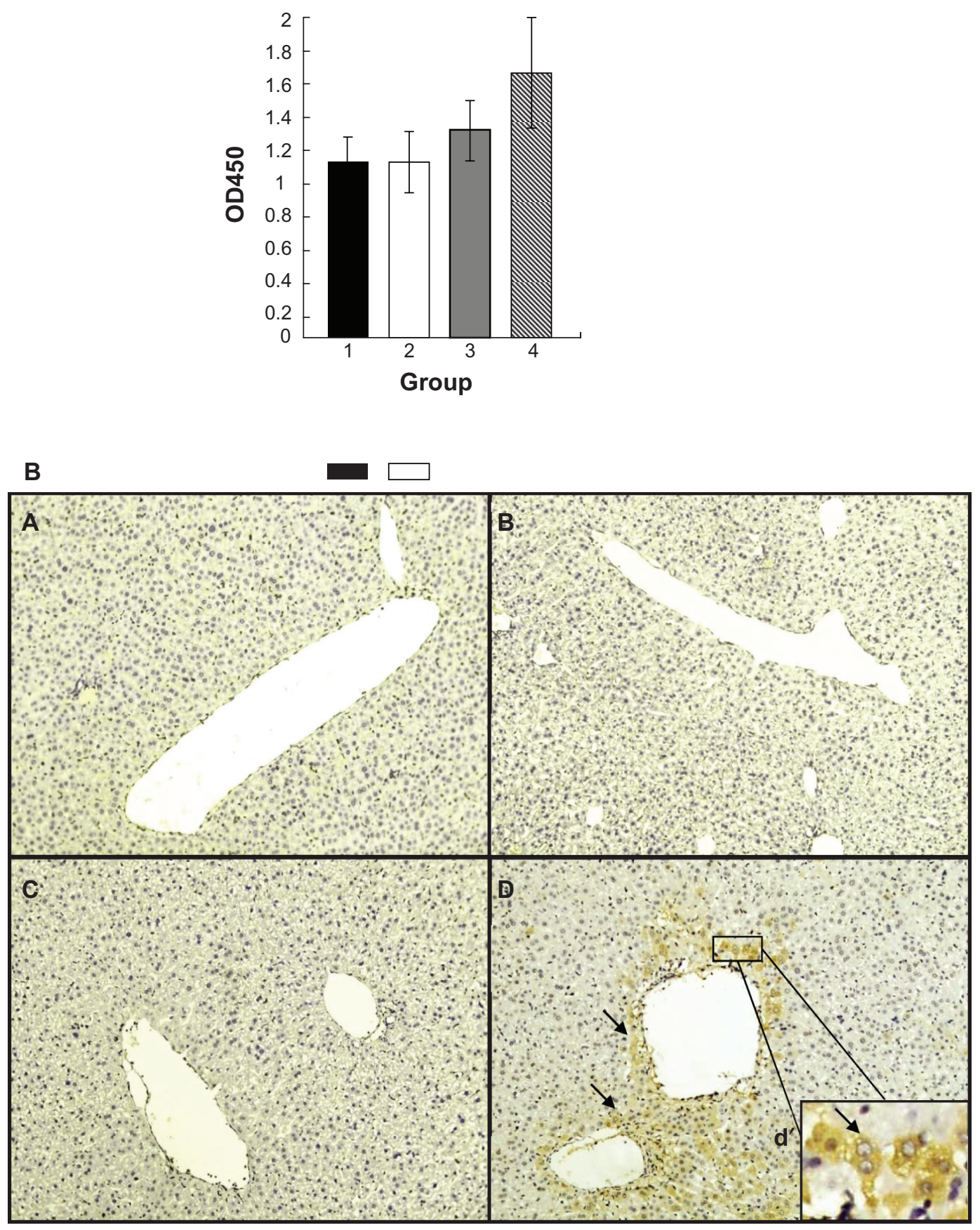

Figure 4 Coexpression of human SCCAI and FTL in mouse liver enhances HBV replication. Plasmids pcDNA3.I (column I in A; a in B), pcDNA3.I-SCCAI (column 2 in A; $b$ in B), pcDNA3. I-FTL (column 3 in A; c in B), or both of pcDNA3. I-SCCAI and pcDNA3. I-FTL (column 4 in A; d in B) were introduced into mouse liver via hydrodynamic injection through tail vein. (A) Serum level of $\mathrm{HBs} A g$ determined 24 hours post virus challenge. (B) Immunohistochemical staining of $\mathrm{HBc} A \mathrm{~g} 48$ hours post virus challenge. Abbreviations: SCCAI, squamous cell carcinoma antigen I; FTL, ferritin light chain; $\mathrm{HBV}$, hepatitis B virus; $\mathrm{HBs} A g$, hepatitis $B$ virus surface protein antigens; $\mathrm{HBcAg}$, hepatitis $B$ virus core protein antigens.

for both baits (preS and SCCA1) in our extensive screening. Several lines of evidence supported our hypothesis that FTL could be a cofactor for SCCA1 in HBV cellular attachment: (1) both preS and SCCA1 are bound to FTL in vitro and in HepG2 cells, (2) preS and SCCA1 bind to distinct subregions of FTL, (3) coexpression of FTL and SCCA1 enhanced HBV infection in human HepG2 and CHO cells, and (4) coexpression of FTL and SCCA1 in mice liver via hydrodynamic injection enhanced HBV attachment and replication in hepatocytes.

Unlike preS1 domain, preS2 domain seems dispensable for HBV infectivity, ${ }^{22}$ but in another study, Le Seyec ${ }^{23}$ 
demonstrated that only the first 5 amino acids at the $\mathrm{N}$-terminal of the preS2 domain is essential for virion export and the preS2 domain could still contribute to virus entry. ${ }^{24}$ In our results, the binding motif of preS2 is located right in the N-terminal 11 amino acids.

Ferritin is the major iron storage protein of mammals with a molecular weight of $450 \mathrm{kDa}$, which consists of 24 heavy or light chains and is expressed in various tissues. Ferritin in liver and serum contains only 24 light chains. ${ }^{25}$ Despite the only 53\% sequence homology, human FTL and ferritin heavy chain (FTH) share an almost identical tertiary structure consisting of five helices. ${ }^{26}$ It is not clear how ferritin is secreted into serum since neither FTH nor FTL have signal peptides which are essential for secretory proteins. One possibility is that the special tertiary structures of FTL and FTH make them easy to fuse with the cell membrane and pass through it so that ferritin is assembled outside the cells. Another possibility is that it is the characteristic global structure of ferritin that makes it easy to pass through the cell membrane. In either case, FTL should be able to fuse with cell membranes and hence directly interact with membrane protein SCCA1 on HepG2 cells.

The deletion experiments revealed that helix $\mathrm{A}$ is the binding site of preS while the other four helices (B-E) are required for maintaining the correct three-dimensional structure needed for binding of SCCA1. We compared the FTL amino acid sequences of human, bovine, and mouse, and found that there are two key amino acid mutations. As shown in Figure 5A, the serine amino acid residue at position 19 of the N-terminus of human FTL is replaced by arginine in mouse and bovine FTL. Similarly, the glutamine residue at position 26 of human FTL is replaced by arginine in mouse and bovine FTL. The two mutations (S19R and Q26R) introduce two positive charges, and this may directly modulate the protein-protein interaction of FTL to preS2, since the side chains of the two amino acids is located at the surface of helix A as predicted by molecular modeling (Figure 5B). The differential surface change may explain host restriction of HBV infection, and no interference of bovine or mouse FTL in our cell culture and mouse studies.

Coreceptors are widely involved in viral infection. Besides the well-known coreceptors CCR5 or CXCR4 that are required for HIV attachment to CD4+ cells, ${ }^{27,28}$ studies on cellular receptors of hepatitis $\mathrm{C}$ virus, ${ }^{29}$ Epstein-Barr virus, ${ }^{30}$ and other viruses have implicated coreceptors in viral infection. Advantages for viruses to have coreceptors for cellular infection rather than a single receptor protein may include avoiding loss of infectiveness due to host gene mutations, and increasing specificity of host cell types.

This work is the first case reporting a successful in vivo infection of HBV in mouse hepatocytes with the help of hydrodynamic injection. However, solid evidence is required to confirm the role of FTL as the second receptor for HBV infection. One possible approach may be preparation of transgenic mice coexpressing human SCCA1 and FTL genes so that the susceptibility to HBV infection of the transgenic mice could be investigated. Nevertheless, our study may shed new light on the nature of HBV infection and may provide new targets for drug development to prevent HBV infection and for

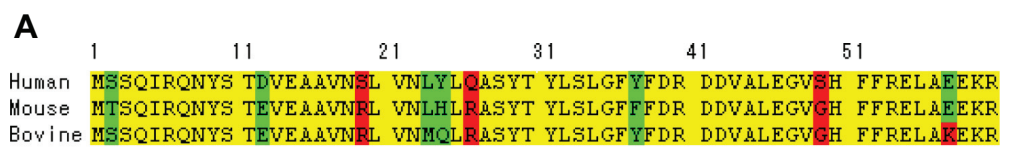

\section{B}

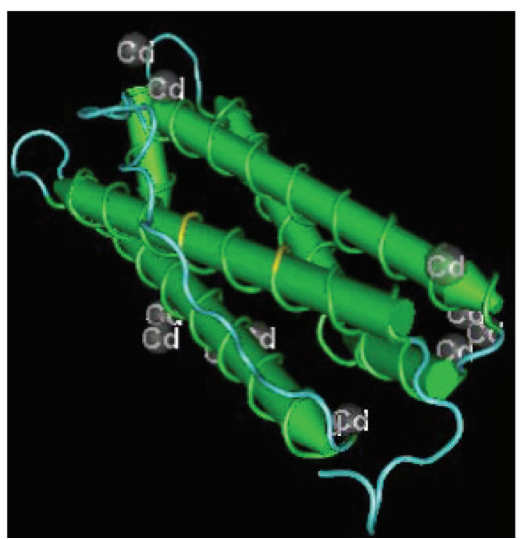

Figure 5 (A) Comparison of the FTL N-terminal amino acids sequence of human, mouse and bovine. (B) Illustration of the position of SI9R and Q26R on 3D structure. Abbreviation: FTL, ferritin light chain. 
therapeutic use. Realistically, infusion of excessive FTL may block HBV infection since expression of FTL in HepG2 cells could reduce the absorption of HBV significantly.

\section{Acknowledgments}

This work is supported by the Shanghai Leading Academic Discipline Project (No. B111), the NSF of China (No. 50533010), and by a grant from the Shanghai Hengda Sciences and Technology Development Co, Ltd, China.

\section{Disclosure}

The authors report no conflicts of interest in this work.

\section{References}

1. Ganem D, Varmus HE. The molecular biology of the hepatitis B viruses. Annu Rev Biochem. 1987;56:651-693.

2. Stibbe W, Gerlich WH. Structural relationships between minor and major proteins of hepatitis B surface antigen. J Virol. 1983;46:626-629.

3. Heermann KH, Goldmann U, Schwartz W, Seyffarth T, Baumgarten H, Gerlich WH. Large surface proteins of hepatitis B virus containing the pre-S sequence. J Virol. 1984;52:396-402.

4. Neurath AR, Kent SB, Strick N, Parker K. Identification and chemical synthesis of a host cell receptor binding site on hepatitis B virus. Cell. 1986;46:429-436.

5. Neurath AR, Seto B, Strick N. Antibodies to synthetic peptides from the PreS I region of the hepatitis B virus (HBV) envelope (env) protein are virus neutralizing and protective. Vaccine. 1989;7:234-236.

6. Paran N, Geiger B, Shaul Y. HBV infection of cell culture: evidence for multivalent and cooperative attachment. EMBO J. 2001; 20:4443-4453.

7. Bruss V, Ganem D. The role of envelope proteins in hepatitis B virus assembly. PNAS. 1991;88:1059-1063.

8. Colucci G, Beazer Y, Waksal SD. Interactions between hepatitis B virus and polymeric human serum albumin. II. Development of syngeneic monoclonal anti-anti-idiotypes which mimic hepatitis B surface antigen in the induction of immune responsiveness. Eur J Immunol. 1987; 17:371-374.

9. Bruin W, Leenders W, Kos T, Hertogs K, Depla E, Yap SH. Hepatitis delta virus attaches to human hepatocytes via human liver endonexin II, a specific HBsAg binding protein. J Viral Hepat. 1994; $1: 33-38$.

10. Treichel U, Meyer zum Büschenfelde KH, Dienes HP, Gerken G. Receptor-mediated entry of hepatitis B virus particles into liver cells. Arch Virol. 1997;142:493-498.

11. Treichel U, Meyer zum Büschenfelde KH, Stochert RJ, Poralla T, Gerken G. The asialoglycoprotein receptor mediates hepatic binding and uptake of natural hepatitis B virus particles derived from viraemic carrier. J Gen Virol. 1994;75:3021-3029.

12. De Falco S, Ruvoletto MG, Verdoliva A, et al. Cloning and expressing of a novel hepatitis B virus binding protein from HepG2 cells. J Biol Chem. 2001;276:36613-36623.

13. Suminami Y, Kishi F, Sekiguchi K, Kato H. Squamous cell carcinoma antigen is a new member of the serine protease inhibitors. Biochem Biophys Res Commun. 1991;181:51-58.

International Journal of Nanomedicine

\section{Publish your work in this journal}

The International Journal of Nanomedicine is an international, peerreviewed journal focusing on the application of nanotechnology in diagnostics, therapeutics, and drug delivery systems throughout the biomedical field. This journal is indexed on PubMed Central, MedLine, CAS, SciSearch $\AA$, Current Contents $₫ /$ Clinical Medicine,
14. Liu F, Song YK, Liu D. Hydrodynamics-based transfection in animals by systemic administration of plasmid DNA. Gene Ther. 1999; 6:1258-1266.

15. Zhang G, Budker V, Wolff JA. High level of foreign gene expression in hepatocytes after tail vein injections of naked plasmid DNA. Hum. Gene Ther. 1999;10:1735-1737.

16. Yang PL, Althage A, Chung J, Chisari FV. Hydrodynamic injection of viral DNA: A mouse model of acute hepatitis B virus infection. PNAS. 2002;99:13825-13830.

17. Neurath AR, Strick N, Sproul P. Search for hepatitis B virus cell receptors reveals binding sites for interleukin 6 on the virus envelope protein. J Exp Med. 1992;175:461-469.

18. Neurath AR, Strick N. Antigenic mimicry of an immunoglobulin A epitope by a hepatitis B virus cell attachment site. Virology. 1990; 178:631-634.

19. Ryu CJ, Cho DY, Gripon P, Kim HS, Guguen-Guillouzo C, Hong HJ. An 80-kilodalton protein that binds to the pre-S1 domain of hepatitis B virus. J Virol. 2000;74:110-116.

20. Pontisso P, Petit MA, Bankowski MJ, Peeples ME. Human liver plasma membranes contain receptors for the hepatitis B virus pre-S1 region and, via polymerized human serum albumin, for the pre-S2 region. J Virol. 1989;63:1981-1988.

21. Xia HB, Chen ZY, Chen XG. Overexpression of hepatitis B virusbinding protein, squamous cell carcinoma antigen 1, extends retention of hepatitis B virus in mouse liver. Acta Biochim Biophys Sin (Shanghai). 2006;38:484-491.

22. Yi N, Jessika S, Stefan S, Stephan U. The pre-S2 domain of the hepatitis $\mathrm{B}$ virus is dispensable for infectivity but serves a spacer function for L-protein connected virus assembly. J Virol. 2010;84:3879-3888.

23. Le Seyec J, Chouteau P, Eannie J, et al. Role of the pre-S2 domain of the large envelope protein in Hepatitis B virus assembly and infectivity. J Virol. 1998;72:5573-5578.

24. Xie Y, Zhai J, Deng Q, Tiollais P, Wang Y, Zhao M. Entry of hepatitis B virus: mechanism and new therapeutic target. Pathol Biol (Paris). 2010;58:301-307.

25. Harrison PM, Arosio P. The ferritins: molecular properties, iron storage function and cellular regulation. Biochim Biophys Acta. 1996;1275:161-203.

26. Wang ZM, Chester Li, Melanie Ellenburg, et al. Structure of human ferritin L chain. Acta Crystallogr D Biol Crystallogr. 2006; 62:800-806.

27. Clapham PR, McKnight Á. HIV-1 receptors and cell tropism. Br Med Bull. 2001;58:43-59.

28. Whitcomb JM, Huang W, Fransen S, et al. Development and characterization of a novel single-cycle recombinant-virus assay to determine human immunodeficiency virus type 1 coreceptor tropism. Antimicrob Agents Chemother. 2007;51:566-575.

29. Bartosch B, Vitelli A, Granier C, et al. Cell entry of hepatitis C virus requires a set of co-receptors that include the CD81 tetraspanin and the SR-B1 scavenger receptor. J Biol Chem. 2003;278:41624-41630.

30. Haan KM, Kwok WW, Longnecker R, Speck P. Epstein-Barr virus entry utilizing HLA-DP or HLA-DQ as a coreceptor. $J$ Virol. 2000;74:2451-2454.

31. Crespo A, Peydro A, Dasi F, et al. Hydrodynamic liver gene transfer mechanism involves transient sinusoidal blood stasis and massive hepatocyte endocytic vesicles. Gene Ther. 2005;12:927-935.

Journal Citation Reports/Science Edition, EMBase, Scopus and the Elsevier Bibliographic databases. The manuscript management system is completely online and includes a very quick and fair peer-review system, which is all easy to use. Visit http://www.dovepress.com/ testimonials.php to read real quotes from published authors. 